\begin{tabular}{|l|l|l|}
\hline \multicolumn{2}{|c|}{ PublisherInfo } \\
\hline \hline PublisherName & $:$ & BioMed Central \\
\hline \hline PublisherLocation & $:$ & London \\
\hline \hline PublisherImprintName & $:$ & BioMed Central \\
\hline \hline
\end{tabular}

\title{
Articles selected in May 2004
}

\begin{tabular}{|l|l|l||}
\hline \multicolumn{2}{|c|}{ ArticleInfo } \\
\hline \hline ArticleID & $:$ & 819 \\
\hline \hline ArticleDOI & $:$ & $10.1186 /$ bcr817 \\
\hline \hline ArticleCitationID & $:$ & E13 \\
\hline \hline ArticleSequenceNumber & $:$ & 28 \\
\hline \hline ArticleCategory & $:$ & Article selection \\
\hline \hline ArticleFirstPage & $:$ & 1 \\
\hline \hline ArticleLastPage & $:$ & 3 \\
\hline \hline & & RegistrationDate : 2004-6-4 \\
ArticleHistory & $:$ & OnlineDate $\quad$ 2004-6-4 \\
\hline \hline ArticleCopyright & $:$ & The Author(s)2004 \\
\hline \hline ArticleContext & $:$ & 130586644 \\
\hline \hline
\end{tabular}


Valerie Speirs, Aff1

Corresponding Affiliation: Aff1

Email: v.speirs@leeds.ac.uk

Aff1 Molecular Medicine Unit, University of Leeds, St James's University

Hospital, Leeds LS9 7TF, UK

\section{Articles selected from PubMed}

\section{References}

1. Jones C, Mackay A, Grigoriadis A, Cossu A, Reis-Filho JS, Fulford L, Dexter T, Davies S, Bulmer K, Ford E, Parry S, Budroni M, Palmieri G, Neville AM, O'Hare MJ, Lakhani SR: Expression profiling of purified normal human luminal and myoepithelial breast cells: identificationof novel prognostic markers for breast cancer. Cancer Res. 2004, 64: 3037-3045. [http://www.ncbi.nlm.nih.gov/entrez/ query.fcgi? $\mathrm{cmd}=$ Retrieve $\& \mathrm{db}=$ pubmed $\&$ dopt $=$ Abstract\&list_uids $=15126339]$

2. Crawford YG, Gauthier ML, Joubel A, Mantei K, Kozakiewicz K, Afshari CA, Tlsty TD: Histologically normal human mammary epithelia with silenced p16(INK4a) overexpress COX-2, promoting a premalignant program. Cancer Cell . 2004, 5: 263-273. [http://www.ncbi.nlm.nih.gov/ entrez/query.fcgi? $\mathrm{cmd}=$ Retrieve $\& \mathrm{db}=$ pubmed\&dopt $=$ Abstract\&list_uids $=15050918]$

3. Uzzan B, Nicolas P, Cucherat M, Perret GY: Microvessel density as a prognostic factor in women with breast cancer: a systematic review of the literature and meta-analysis. Cancer Res. 2004, 64: 2941-2955. [http://www.ncbi.nlm.nih.gov/entrez/ query.fcgi? $\mathrm{cmd}=$ Retrieve $\& \mathrm{db}=$ pubmed $\&$ dopt $=$ Abstract\&list_uids $=15126324]$

4. Sun SY, Hail N, Lotan R: Apoptosis as a Novel Target for Cancer Chemoprevention. J Natl Cancer Inst. 2004, 96: 662-672. [http://www.ncbi.nlm.nih.gov/entrez/ query.fcgi?cmd $=$ Retrieve $\& \mathrm{db}=$ pubmed $\&$ dopt $=$ Abstract\&list_uids $=15126603$ ] 
5. Hashimoto S, Onodera Y, Hashimoto A, Tanaka M, Hamaguchi M, Yamada A, Sabe H: Requirement for Arf6 in breast cancer invasive activities. PNAS. 2004, 101: 6647-6652.

[http://www.ncbi.nlm.nih.gov/entrez/

query.fcgi? $\mathrm{cmd}=$ Retrieve $\& \mathrm{db}=$ pubmed $\&$ dopt $=$ Abstract\&list_uids $=15087504]$

6. Cardonick E, Iacobucci A: Use of chemotherapy during human pregnancy. Lancet Oncol. 2004, 5:

283-291. [http://www.ncbi.nlm.nih.gov/entrez/

query.fcgi? $\mathrm{cmd}=$ Retrieve $\& \mathrm{db}=$ pubmed $\&$ dopt $=$ Abstract\&list_uids $=15120665]$

This PDF file was created after publication. 